\title{
Die Impfung gegen die Tuberkulose der Rinder.
}

\author{
Nach einem Bericht, erstattet zum IX. Internationalen Tierärztl. \\ Kongress im Haag 1909 \\ von
}

Prof. Dr. M. Klimmer, Dresden.

In nachfolgenden Ausführungen habe ich nur diejenigen Impfverfahren gegen die Tuberkulose der Rinder berücksichtigt, welche bisher Eingang in die tierärztliche Praxis gefunden haben. Es sind das folgende:

1. Die Bovovakzination v. Behrings. Vorwiegend niedergelegt in den Beiträgen zur experimentellen Therapie, herausgeg. von v. Behring, H. 5-10, und den Behringwerk-Mitteilungen Heft 1-2.

Impfstoff: Getrocknete Menschentuberkelbazillen (Bovo vakzin). Dieselben sind am Orte der Impfung mit sterilisiertem Wasser zu einer Emulsion zu verreiben. Zur ersten Impfung erhalten die zu impfenden 2-12 Wochen alten Kälber $4 \mathrm{mg}, \mathrm{zu}$ der ein Vierteljahr später vorzunehmenden zweiten Impfung $20 \mathrm{mg} \mathrm{Bovovakzin} \mathrm{in} \mathrm{die} \mathrm{Vena} \mathrm{jugularis} \mathrm{eingespritzt.} \mathrm{Weitere} \mathrm{Impfungen}$ werden nicht vorgenommen. Besondere hygienische Massnahmen sind nicht vorgeschrieben.

2. Die Impfung mit Tauruman nach Koch und Seh ïtz (Neufeld nnd Miessner), voröffentlicht im Archiv für wissenschaftliche und praktische Tierheilkunde 1905, Band 31, Seite 545 und Zeitschr. f. Hyg. a. Infektions-Krankh. Bd. 51 , S. 300.

Impfstoff: Menschentuberkelbazillen in wässeriger Aufschwemmung ( $\mathrm{T}$ a u ruman). Dosis: $10 \mathrm{ccm}$ ( $\mathrm{mg} \mathrm{10)}$; einmalige intravenöse Impfung von Kälbern im Alter von ca. 3 Monaten. Besondere hygienische Massnahmen sind nicht vorgeschrieben.

3. Das Heymanssche Verfahren. Arch. internat. de Pharmacodynamie et de Thérapie. 1905, Vol. 14, p. 171 und 1908, Vol. 18, p. 179.

Impfstoff: In Schilfsäckchen eingeschlossene, getrocknete Menschentuberkelbazillen, welche ausserdem noch von einer Gelatinekapsel umgeben sind. 
Die Impfung wird an Rindern jeden Alters und Geschlechtes als Schutz- und Heilimpfung mit Hilfe eines Troikards in das Unterhautbindegewebe bewirkt und ist jährlich zu wiederholen. Besondere hygienische Massnahmen finden anscheinend keine Anwendung.

4. Das Tuberkulosetilgungsverfahren mit Hilfe des nicht infektiösen Impfstoffes Antiphymatol nach $\mathbf{K} \mathbf{l}$ imm e r, vornehmlich mitgeteilt in den Berichten über das Veterinärwesen im Königreich Sachsen bezw. der Königlichen Tierärzllichen Hochschule zu Dresden für das Jahr 1903 und ff., der Zeitschrift für Tuberkulose, Band 12, Heft 5/36, Zeitschrift für Tiermedizin, Band 12 p. 81 und der Deutschen Tierärztlichen Wocbenschrift, 1909, p. 1.

Impfstoff: Avirulente ${ }^{1}$ ) Tuberkelbazillen in wässeriger Aufschwemmung (Antiphymatol). Dosis: $5 \mathrm{ccm}$. Die Impfung wird an Rindern jeden Alters und Geschlechts vor allem als Schutz- und in zweiter Linie als Heilimpfung in das Unterhautbindegewebe, also nicht wie bei den unter 1 und 2 genannten Verfahren intravenös, bewirkt und zwar bei tuberkulosefreien Tieren im ersten Jahre zweimal, bei tuberkulösen viermal in einvierteljährlichen Pausen. Später in jedem Jahre einmalige Nachimpfung.

Neben der Impfung, der möglichst alle Rinder, zum mindesten die gesamte junge Aufzucht und die tuberkulosefreien Rinder zu unterziehen sind, sollen die Tiere vor einer Milchinfektion nach Möglichkeit geschützt werden (tuberkelbazillenfreie soust pasteurisierte Milch oder wenigstens Wilch nur einer möglichst gesunden Kuh und keine Mischmilcb mehrerer Kühe). Es wird empfohlen, zu Be. ginn der Tuberkulosetilgung in einem Bestande die Rinder einer Tuberkulinprobe (Ophthalmoreaktion mit wirksamem Tuberkulin ${ }^{2}$ ) zu unterwerfen; die nicht reagierenden Tiere werden in geschlossener Reihe aufgestellt und ihnen, wenn sie in Doppelreihen mit den Köpfen gegenüber stehen, nach Möglichkeit nicht reagierende Tiere gegenüber angebunden. Ferner sind die Tiere auf Eutertuberkulose schon aus sanitären Gründen zu untersuchen und eutertuberkulös befundene, desgleichen chronische Huster (Tiere mit offener Lungentuberkulose) baldigst ab. zuschlachten.

Bei der Beurteilung obiger Impfverfahren gegen die Tuberkulose kommen rorwiegend folgende Punkte in Frage:

1. ihre Ungefährlichkeit für Menschen.

a) beim Impfakt,

b) bei der Wartung der Impflinge,

c) hinsichtlich des Genusses ron Fleisch und Milch geimpfter Tiere.

2. ihre Ungefährlichkeit für die Impflinge.

3. ihre Wirksamkeit.

1) Die avirulenten $\mathrm{Tb}$ wurden aus den Organen von Molchen herausgezüchtet, die wiederholt mit Menschentuberkelbazillen, die mehrfache Molchpassagen durchgemacht hatten, behandelt waren. Wie schon im Namen ausgedrückt ist, sind sie avirulent und zwar für Menschen, Säugetiere, Vögel und Kaltblüter.

2) Die zuverlässigsten Ergebnisse gibt das von der chemischen Fabrik H u m an n und Teisler, Dohna b. Dresden, für die Ophthalmoreaktion besonders hergestellte Phymatin. Klimmer im Handbuch der Serotherapie, herausgegeben von Wolff-Eisner und Klimmer, Lejpzig 1911. 


\section{Welche Gefahren bieten die verschiedenen Tuberkulose- impfverfahren für Menschen?}

Bei der Beurteilung der verschiedenen Impfverfahren gegen die Tuberkulose der Rinder sind die Gefahren, welche sich bei oder aus der Impfung der Rinder $f \ddot{u} r$ M enschen ergeben können, sehr wohl mit zu berücksichtigen. Der Schutz der Rinder gegen die Tuberkulose wäre schon zu teuer, wenn er mit der Gesundheit oder gar dem Leben auch nur eines Menschen bezahlt würde. In erster Linie muss somit bei der Beurteilung der verschiedenen Impfrerfahren, bei denen mit menscheninfektiösen Tuberkelbazillen gearbeitet wird, geprüft werden, ob auch der oberste, leider nur zu oft nicht genügend beobachtete Grundsatz der Medizin "Non noceat" auch sicher erfüllt ist und ganz besonders soweit, als der Mensch hierbei in Frage kommt. Für den Menschen können selbstverständlich nur solche Tuberkuloseimpfstoffe gefährlich werden, welche virulente Tuberkelbazillen enthalten. Die Virulenz der hier als Impfstoffe in übereinstimmender Weise verwendeten Nenschentuberkelbazillen pflegt man am Meerschweinchen zu prïfen und aus den hierbei gewonnenen Ergebnissen, wenn sie ein Fehlen der Infektiosität erbracht haben, zu schliessen, dass die betreffenden Impfstoffe dann auch für Menschen nicht mehr virulent sind. Um klare, eindeutige Resultate zu erhalten, empfiehlt es sich, das zu prüfende Material in die Muskelmasse des Hinterschenkels einzuspritzen und die Dosis des Impfstoffes etwa so zu wählen, dass darin etwa 1-2 mg Tuberkelbazillen enthalten sind.

Bei der Prüfung der hier in Frage kommenden Impfstoffe hat sich ergeben, dass der B o vovakzinv. Behrings zumeist (- nach v. Behring (Behringwerk-Mitteilungen Heft 2, S. 48) "besitzt er einen solchen mittleren Virulenzgrad, dass mit $1 \mathrm{~g}$ von meinem Bovovakzin ca. 10000 Meerschweinchen mit tötlichem Ausgang infiziert werden" -), das Tauruman Koch-Schützs stets und der Hèymanssche Impfstoff wohl ebenfalls immer meerschweinchenvirulente Tuberkelbazillen enthalten, dagegen ist der nicht-infektiöse Tuberkuloseimpfstoff Klimmers, das Antiphymatol, frei von meerschwein-virulenten Tuberkelbazillen (Schnürer, $\mathrm{H}$ a mburger, Bandelier etc.) und erlangt auch durch 1, 2, 4, 10 und 20 Wochen langes Verweilen im Säugetierkörper (Kaninchen, Rind) keine Virulenz für Meerschweinchen. Wenn auch schon aus diesen Tierversuchen mit an Sicherheit grenzender Wahrscheinlichkeit geschlossen werden kann, dass das Antiphymatol keinerlei Gefahren für Menschen sowohl beim 
Impfakt als auch hinsichtlich des Konsums von Fleisch und Milch der Impflinge bietet, so ist diese überaus wichtige, sanitäre Frage des weiteren noch durch bereits ca. 500 therapeutische und prophylaktische Impfungen direkt an Menschen dahin beanwortet worden, dass er auch nicht menschenvirulent ist (Bandelier, Plettner etc.).

Bei den drei anderen Impfstoffen (Bovovakzin v. Behrings, Tauruman Koch-Schützs und Impfstoff Heymans), welche meerschweinchenvirulente Tuberkelbazillen meist oder stets enthalten, kommt hinsichtlich der Gefahren, die sie bei den Arbeiten oder dem Konsum von Fleisch und Milch von Impflingen bieten, des weiteren ihr Virulenzgrad in Frage. Soweit in dieser Richtung Mitteilungen vorliegen, ist das Tauruman stärker virulent für Meerschweinchen als der Bovovakzin. Über den Virulenzgrad des in Schilfsäcken eingeschlossenen Heymansschen Impfstoffes scheinen keine Angaben vorzuliegen.

Da die Virulenz für Meerschweinchen wohl noch nicht ohne weiteres mit einer solchen $f \ddot{u r}$ Menschen identifiziert werden kann, so ist auf letztere noch mit wenig Worten einzugehen.

Dass das Tauruman eine sehr beträchtliche Virulenz für Menschen besitzt, geht aus der Mitteilung von Kreistierarzt Möller (Deutsche Tierärztliche Wochenschrift 1906, S. 631) hervor.

Möller erwähnt, dass sein Assistent, Tierarzt W., beim Impfen der Kälber mit Koch-Schützschen Tauruman sich einmal eine etwas leichtere, ein anderes Mal eine sehr schwere Tuberkuloseinfektion zuzog. Im ersteren Fall erinnert sich W. nicht, sich verwundet zu haben, während er im letzteren Falle Schürfwunden an der linken Hand batte.

Hinsichtlich des Virulenzgrades des Bovorakzins r. Behrings für Menschen gehen die Mitteilungen in gleicher Weise auseinander, wie bezüglich jener für Meerschweinchen. Es muss hiernach angenommen werden, dass der Bovovakzin ein sehr ungleiches Produkt ist. Während Ebeling sich nach einer Mitteilung v. Behrings versehentlich und wenigstens zunächst ohne Schaden etwas ron e ine $r$ Bovovakzinaufschwemmung in die Hand eingespritzt hat, berichtete $\mathrm{Hagem}$ a $\mathrm{n}$ auf der Versammlung deutscher Naturforscher und Ärzte in Dresden 1907 in der Sektion für praktische Tierheilkunde, dass er sich bei der Impfung der Kälber mit dem Bovovakzin v. Behrings eine Infektion am Finger zuzog und deshalb diese Impfmethode später verliess. Hiernach besteht die $G$ efahr einer Infektion von Menschen durch den Borovakzin v. Behrings.

Der Heymanssche Impfst off enthält meerschweinvirulente Tuberkelbazillen. Da diese Bazillen menschlichen Ursprungs und 
ausser dem Trocknungsprozess einem besonderen Abschwächungsverfahren wohl nicht unterworfen worden sind, so dürften die Bazillen wohl ebenfalls menschenvirulent sein. In der einschlägigen Literatur sind mir Angaben über stattgefundene Infektionen des Menschen beim Impfakt nicht bekannt. Hervorzuheben ist hier noch, dass die Tuberkelbazillen im $\mathrm{H} \in \mathrm{ym}$ ansschen Impfstoff in Schilfsäckchen und diese ausserhalb des Tierkörpers von Gelatinekapseln, welche im Tierkörper bald aufgelöst werden, eingeschlossen und dadurch vor einer Zerstreuung und Verbreitung im Tierkörper solange geschützt sind, als die Säckchen dicht sind.

\section{a) Gefahren beim Impfakt.}

Die Impfstoffe, welche virulente Tuberkelbazillen enthalten, können beim Impfakt insofern zur Tuberkuloseübertragung auf Menschen Anlass geben, als der Impftierarzt oder seine Umgebung sich eine Wundinfektion mit virulenten Tuberkelbazillen zuziehen kann. Wie schon an Beispielen gezeigt wurde, sind solche beim Bovovakzin und Tauruman vorgekommen.

Der Bovovakzin v. Behrings kann möglicherweise auch dadurch dem Menschen gefährlich werden, dass der aus getrockneten Menschentuberkelbazillen bestehende Impfstoff bei dem A r beiten (Ausschütten, Abwägen, Verreiben etc.) teilweise verstäubt oder verspritzt und mit der Atmungsluft aufgenommen oder mit infizierten und hiernach nicht hinlänglich desinfizierten Händen etc. eventuell unter Vermittlung von Nahrungsmitteln etc. auf Menschen übertragen wird.

In Hinblick darauf, dass der Bovovakzin Wundinfektionen beim Menschen hervorrufen kann, erscheint mir auch eine Infektion vom At mungs- oder Verdauungapparat aus dann nicht völlig ausgeschlossen, wenn das Arbeiten mit diesen getrockneten Menschentuberkelbazillen ohne weitgehende Sicherheitsmassregeln vorgenommen wird. Soweit ich einen Einblick gewonnen habe, pflegt man mit dem Bovovakzin ohne besondere Kautelen umzugehen. In dem meist chronischen Verlauf der menschlichen Tuberkulose und den meist sehr vielfachen anderen Ansteckungsmöglichkeiten liegt es begründet, dass ein einwandsfreier Beweis für eine durch das Arbeiten mit dem Bovovakzin veranlasste Tuberkuloseübertragung auf den Atmungs- und Verdauungsapparat nicht erbracht werden kann.

Da das flüssige Ta urum an gebrauchsfertig abgegeben wird, ist die Gefahr der Versträuung von Tuberkelbazillen wesentlich geringer, wenn auch eine Übertragung mit infizierten Händen etc. nicht völlig ausgeschlossen ist. 
Der sorgfältig hergestellte Heymans sche Impfstoff, bei dem die Tuberkelbazillen zunächst in Schilfsäckchen und diese wiederum in Gelatinekapseln eingeschlossen sind, dürfte dagegen, wenn nicht sehr grobe Versehen sich ereignen, bei den Impfarbeiten als ungefährlich anzusehen sein.

Gefahrlos ist, wie schon betont, das Antiphymatol.

b) Gefahren bei der Wartung und Pflege der Impflinge.

Die mit virulenten Tuberkelbazillen geimpften Tiere können bei der Wartung und Pflege für das Stallpersonal gegebenen Falles dadurch gefährlich werden, dass sie mit den Exkreten (auf die Sekrete speziell die Milch soll später eingegangen werden) Menschentuberkelbazillen a usscheiden, die auf verschiedene Weise ibren Weg in den Menschen finden können. Sodann kann es sich auch ereignen, dass der v. Behringsche und Koch-Schiitzsche Impfstoff trotz aller Übung in der Technik der intravenösen Injektionen bei Unruhe des Tieres etc. versehentlich unter di e $\mathrm{Haut}$, bezw. in das lockere, die Vena jugularis umgebende Bindegewebe gelangt und im weiteren Verlaufe - und zwar gilt dies ganz besonders von dem Tauruman - zur Bildung eines tuberkulösen Abszesses Anlass gibt, der vielfach aufbricht und zur Versträung von darin enthaltenen rirulenten Menschentuberkelbazillen führt (Weber und Titze, Schütz und Holland, Tuberkulose-Arbeiten aus dem Kais. Gesundheitsamte H. 9, S. 32). Bei dem Heymansschen I mpist off könnte dies nur dann eintreten, wenn zuvor das Schilfsäckchen zerreisst. Mitteilungen über das Auftreten tuberkulöser Abszesse nach der Impfung nach $\mathrm{Heymans}$ liegen in der Literatur nicht vor.

Gefahrlos ist in dieser Richtung wiederum das Antiphymatol.

\section{c) Gefahren durch den Genuss von Fleisch und Milch geimpfter} Tiere.

Bei der Beurteilung der Gefahr, die sich aus dem Genuss von ungenügend erhitztem Fleisch und Milch von Impflingen ergeben kann, ist man auf die Ergebnisse von Meerschweinchenversuchen an. gewiesen.

Die Frage der Haltbarkeit menschlicher 'Tuberkelbazillen im Körper des Rindes haben zunächst einmal II affucci und Lignières geprüft. Ersterer fand (La clinica moderna 1903 Nr. 34), dass menschliche Tuberkelbazillen nach subkutaner Einspritzung in den regionären Lymphdrüsen bis zu 8 Monaten am Leben und infektionstiichtig bleiben können. 
Nach den Vitteilungen Ligni ères auf dem VIII. internationalen tierärztlichen Kongress zu Budapest 190 ó konnten subkutan auf das Rind rerimpfte menschliche Tuberkelbazillen noch nach 2 Jahren an der Impfstelle und in den benachbarten Drüsen nachgewiesen werden.

Diese Frage der Haltbarkeit menschlicher Tuberkelbazillen im Rinderkörper ist vor allem von Weber und Titze, Schütz und $\mathrm{H}$ olland (Tuberkulose-Arbeiten aus dem Kaiserl. Gesundheitsamte 1908, Heft 9, Seite 27) eingehend studiert worden.

Weber und Tietze, Schütz und Holland fanden, dass die Tuberkelbazillen des Taurumans nach ivtravenöser Einspritzung im Blute schon nach 8 Tagen verschwunden sind. Nach einem Monat gelang im Meerschweinchenversuch der Nachweis von Tuberkelbazillen noch in Lunge, Bronchial- und Msdiastinaldrüsen, Milz, Nieren, Nierendrüsen, Leber, Portaldrüsen, Retropharyngealdrüsen, oberen, mittleren und unteren Halsiymphdrüsen, Bugdrüsen, Achseldrïsen, Kniefaltendrüsen, Kniekehlendrüsen, medialen Darmbeindrüsen, Impfstelle, VInskulus Psoas, Knochenmark, dahingegen nicht im Blute, Musculus Pectoralis maior und $Z$ werchfellp feiler.

Zwei Monate nach der Impfung wurden menschliche Tuberkelbazillen noch nachgewiesen in Lunge, Bronchial- und Mediastinaldrüsen, Milz, Portal-, Nieren-, Achsel- und Kniefaltendrüsen. Nach 3 Munaten konnten die Tuberkelbazillen nur noch in Lunge, Bronchial- und Mediastinaldrüsen gefunden werden. Hier hielten sie sich bis zum 6. Monat nach der Impfung. 7 Monate nach der Impfung fiel der Nachweis dann negativ aus, wenn die Taurumanimpfung vorschriftsmässig intravenös ausgeführt war. Wurde jedoch ein geringer Teil deз Impfstoffes gleichzeitig unter die Haut am Halse eingespritzt, so waren virulente Tuberkelbazillen noch nach 7, nicht aber mehr nach 9 Monaten an der Impfstelle nachzuweisen, und wurde die ganze Taurumandosis unter die Haut am Halse gespritzt, so gelang der Nachweis von Tuberkelbazillen an der Impfstelle im Meerschweinchenversuch noch nach 11 Monaten 11 Tagen. Jedoch erkrankten von 4 geimpften Meerschweinchen nur noch 2.

Bei der Prüfung der Bovovakzinbazillen auf ihre Haltbarkeit im Rinder. körper konnte Weber und Titze dieselben im Meerschweinchenversuch nachweisen 8 Tage nach der Impfung in der Lunge, Bronchial- und Mediastinal- und mittleren Halsdrïsen; 3 Wochen nach der Impfung in Lunge, Bronchial- und Mediastinal, Portal. und Nierendrüsen, sowie in der Galle und endlich $3^{1}{ }_{2}$ Monate nach der Impfung wiederum in Lunge, Bronchial- und Mediastinaldrüsen, ferner in den Nieren- und Bugdrüsen. Das Ergebnis dieser Versuche zeigt keine so grosse Gesetzmässigkeit wie jene der Taurumanversuche. Dies ist insofern nicht verwunderlich, als der v. Behring sche Impfstoff ein in seiner Zusammensetzung sehr wechselndes Präparat ist.

Auf Grund dieser Versuchsergebnisse wurden vom Kaiserlichen Gesundheitsamte zu Berlin über die Behandlung des Fleisches schutzgeimpfter Tiere folgende Vorschläge angenommen:

1. Lunge und Herz von mit lebenden Tuberkelbazillen immunisierten Rindern sind 10 Monate lang nach der Impfung untauglich.

2. Finden sich Veränderungen an der Impfstelle, so ist die Impfstelle und ihre Umgebung bis einschliesslich der zugehörigen Lymphdrïse untauglich.

3. Der ganze Tierkörper mit Ausnahme von Lunge und Herz ist innerhalb der ersten 4 Monate nach der Imnfung bedingt tauglich. 
Die Ausscheidungron Tuberkelbazillen mit der Milch nach Einspritzung derselben ir die Blutbahn und das Gewebe unter die Haut ist ebenfalls wiederholt Gegenstand eingehender Untersuchungen gewesen.

Web $\theta \mathrm{r}$ berichtet (Tuberkulose-Arbeiten aus dem Kaiserlichen Gesundheitsamte Heft 7), dass eine Kuh auf eine einmalige intravenöse und dreimalige subkutane Einspritzung von menschlichen Tuberkelbazillen letztere bis etwa 16 Monate nach der letzten Impfung aus dem einen anfangs klinisch unveränderten, nach 11 Monaten jedoch nachweishar erkrankten Euterviertel ausschied.

Auch Titze (Tuberkulose-Arbeiten des Kaiserlichen Gesundheitsamtes Heft 9) fand, dass menschliche Tuberkelbazillen, die Milchkühen in die Blutbahn eingespritzt sind, mit der Milch in der Regel ausgeschieden werden. Dio Ausscheidung kann schon 24 Stunden nach der Einspritzung beginnen und zirka 16 Monate lang anhalten. Bei 61 , etwa 8 Jahre alten Kühen, welche nach dem v. Behringschen Verfahren im frühen Lebensalter schutzgeimpft waren, konnten Tuberkelbazillen in der Milch jedoch nicht nachgewiesen werden.

Dagegen erwähnt Bongert (Verhandlungen der Gesellschaft Deutscher Naturforscher und Ärzte. 79. Versammiung Dresden 1907. 2. Teil. 2. Hälfte. S. 559), dass er in der Milch zahlreicher, in der Jugend nach v. Behring geimpfter Kühe bei der Verimpfung auf Meerschweinchen Taberkelbazillen festgestellt habe. B ongert meint: „Ein derartig hoher Prozentsatz von Kïhen, die mit der Milch Tuberkelbazillen ausscheiden, kann nur auf die sogenannte Immunisierung der Nachzucht zurückgeführt werden." Seine Mitteilung lässt jedoch die für vorliegende Frage sehr wichtige Angabe vermissen, welcher Tuberkelbazillen. typus mit der Milch ausgeschieden wurde.

Nach den mitgeteilten Untersuchungen muss es vom sanitären Standpunkte aus als höchst bedenklich bezeichnet werden, wenn Tiere, welche älter sind, als es die ron v. Behring und Koch-Schütz erlassenen Vorschriften zulassen, der Impfung mit Bovovakzin und Tauruman unterzogen werden. Ob die Impfung von weiblichen Rindern im jugendlichen Alter die Gefahr einschliesst, dass diese Tiere im späteren Alter Tuberkelbazillen mit der Milch ausscheiden können, wie dies Bongert behauptet, bedarf in Hinblick auf die Ergebnisse Titzes wohl mindestens noch weiterer Nachprüfung, der vor allem auch solche Tiere mit zu unterziehen sind, bei denen die genannten Impfstoffe in die Subkutis gelangt sind.

Im Torstehenden wurden lediglich der Bovovakzin und das Tauruman berücksichtigt und es ist diesbezüglich zunächst auch auf den Heymansschen Impfstoff einzugehen. Die Tuberkelbazillen die nach dem Heymansschen Verfahren subkutan verimpft werden, sind durch das Schilfsäckchen, - die Gelatinekapsel löst sich bekanntlich im Tierkörper schnell auf - in das sie eingeschlossen sind, zunächst an einem $\ddot{\mathrm{C}}$ bertritt in den Lymph- und Blutstrom gehindert, sie können nicht in die Organe eindringen oder mit der Milch ausgeschieden werden. Selbstrerständlich wird dies nur solange der Fall 
sein, als das Säckchen noch vollkommen dicht ist. Der springende Punkt in der hier vorliegenden Angelegenheit gipfelte somit in der Frage, ob das ausserhalb des Tierkörpers so leicht zerreissliche Schilfsäckchen auch im Unterhautbindegewebe des Körpers gröberen, mechanischen Einwirkungen, wie es treffenden Hornstössen, dem Druck beim Reiben der Tiere an Pfosten, wozu das Juckgefühl in der Wunde zuweilen Anlass geben dürfte etc. mit absoluter Sicherheit $z u$ widerstehen vermag, ohne irgendwie undicht zu werden, sodass der Heymanssche Impfstoff auch Milchkühen, wie es Heymans empfiehlt, ohne jedes sanitäre Bedenken unter die Haut einverleibt werden kann.

Heymans spricht sich in dieser Richtung nicht dahin aus, dass das Schilfsäckchen im Tierkörper unter allen Umständen dicht bleibt, sondern er sagt: "(Archives internat. de Pharmacodynami et de Thérapie Band 18, S. 180) Le sac de roseau placé chez la bête bovine est d'ordinaire trouvé intact à l'autopsie“. Er erwähnt weiter, dass er die Bazillen ausschliesslich nur im Säckchen und nicht in der enzystierenden Membran und den regionären Lymphdrüsen gefunden habe.

Hinsichtlich des Antiphymatols wurde schon auf Seite 3 u. 4 hingewiesen, dass dasselben weder menschen- noch meerschweinvirulent ist und auch nach einfachen 1,2, 4, 10 und 20 Wochen langen Tierpassagen nicht wieder infektiös wird. „Denn wenn der Impfstoff von vornherein keine meerschweinchenvirulenten Tuberkelbazillen enthält, können natürlich solche auch nicht im Körper der damit geimpften Rinder durch den Meerschweinchenversuch nachgewiesen werden", betonen Weber, Schütz, Titze und Holland in ihrer Arbeit „Versuche über die Haltbarkeit der behufs Immunisierung eingespritzten menschlichen Tuberkelbazillen im Körper des Rindes" (Tuberkulose-Arbeiten aus dem Kaiserlichen Gesundheitsamte Heft 9, Seite 31), und zwar beziehen sie diesen Satz auf gewisse Bovovakzinpräparate, er hat aber auch hinsichtlich des Antiphymatols Klimmers volle Gültigkeit.

Der Antiphymatolbazillus wird im Tierkörper sehr bald (4 Tagen) abgetötet. (K li m m e r, Zeitschrift für Tiermedizin. Band 12, S. 119). Eine Gefahr hinsichtlich des Konsums von Fleisch und Milch solcher Tiere, welche mit Antiphymatol behandelt worden sind, besteht daher in keiner Weise, wie das auch von $\mathrm{Schnürer}$ betont wird.

Vorstehende Ausführungen über die Gefahren, welche die Impfung der Rinder gegen Tuberkulose mit den verschiedenen in die Praxis eingefiihrten Impfstoffen für Menschen bieten kann, lassen sich wie folgt zusammenfassen: 
1. Die Durchführung der Impfung mit Bovovakzin und Tauruman ist fïr den Impftierarzt, seine Umgebung, sowie das Stallpersonal nicht ungefährlich.

Die Lunge mit ihren regionären Lymphdrüsen kann nach der Impfung der Rindermit Bovovakzin etwa 4 Monate, nach der Einspritzung von Tauruman ca. 6 Monate lang virulente, menschliche Tuberkelbazillen enthalten. In den Körperlymphdrüsen, mit AusnahmederzurImpfstellegehörigen Lymphdrüsen, konnten virulentemenschliche Tuberkelbazillen etwa 4 Monate lang nach der Impfung noch nachgewiesen werden. Ist der Bovovakzin oder das Tauruman ganz oder teilweise unter die Hautgelangt, so w urdenvirulente menschliche Tuberkelbazillen in den regionären Lymphdrüsenetwa 5 Monate, an der Impfstelle selbst ca. 1 Jahr nach der Impfung gefunden.

Inwieweit die Milch von Impflingen, welche, wiedie Vorschrift sagt, in der Jugend mit Bovorakzin oder Tauruman geimpft worden sind, virulente menschliche Tuberkelbazillen enthalten kann, bedarf noch weiterer Lntersuchungen. Sicher ist es jedenfalls, dass sehr häufig und lange Zeit virulente menschliche Tuberkelbazillen mit der Milch ausgeschieden werden, wennentgegen den Vorschriften ältere weibliche Rinder der Impfung mit Bovorakzin oder Tiluruman nnterzogen werden.

2. Der Heymanssehe Impfstoff dì rfte beim Impfakt im allgemeinen ungefährlich sein. Eine Gefahr hinsichtlich des Genusses von Fleisch und Milch der Impflinge besteht, solange das die virulenten Tuberkelbazillen einschliessende Schilfsäckchen dicht ist, nicht, wohl aber, wenn das Säckchenzerreisst.

3. Das Antiphymatol enthält keinevirulenten Tuberkelbazillen, siekönnen somit auch wederbeim lmpfakt, noch bei der Pflege und Wartung der Impflinge, noch hinsichtlich des Konsums ron Fleischund Milch mit diesen ImpfstoffengejmpfterTiere irgendwelcheGefahren für die menschliche Gesundheit bieten. 


\section{Welche Gefahren bieten die verschiedenen Impfverfahren für die Impflinge.}

Die intravenöse Impfung der Kälber mit dem Bororakzin wird zumeist gut rertragen; sie ist aber für die Impflinge nicht immer völlig ungefährlich.

v. Behring erwähnt, dass „nicht selten mehrtägiges Fieber, zuweilen mit Abnahme von Fresslust" auftritt. Wiederholt sind Ohnmachtsanfälle, Husten und Lungenentzündungen, sogenanntes Akutwerden latenter Kälberpneumonie, beobacbtet worden; einmal verendeten von 40 geimpften nicht weniger als 7 Stück an Lungenentzündung ( $\mathrm{Marks}$ in Beiträgen zur experimentellen 'Therapie, herausgegeben von v. Behring, Heft 10 , Seite 16 a). Vielfach sind die Lungenentzündungen meiner Überzeugung nach direkt durch die Impfung mit dem Bovovakzin hervorgerufen worden und es hat sich mehrfach nicht nur um ein sogenanntes Akutwerden latenter Kälberpneumonie gehandelt, wie dies von verschiedenen Seiten hingestellt wird. Zum Beweise meiner Annahme führe ich an, dass in verschiedenen Gehöften ausschliesslich nur die geimpften, nicht aber die ungeimpft gelassenen Kälber erkrankten, was aber m. o. w. ebenfalls hätte der Fall sein müssen, wenn es sich um die erwähnte Infektionskrankheit gehandelt lä̈tte.

Insgesamt sind nach den Zusammenstellungen $R$ ömers (Bericht über den VIII. Irternat. Tierzärztlichen Kongress Bd. 3, Seite $74 \mathrm{ff}$ ) von 5576 geimpften Rindern $41=0,730 \%$ im direkten Anschluss an die Impfung verendet, eine recht hohe Verlustziffer gegenüber der völligen Unschädlichkeit der Heymansschen und Klimmer schen Impfung.

Nach der Impfung mit Tauruman sind die Kälber nach den spärlichen Mitteilungen in der Literatur häufig m. o. w. stark an Fieber und nicht selten an Lungenentzündung erkrankt, denen sie zuweilen erlegen sind. Auch diese Lungenentzündung führe ich mindestens zu einem nicht unbedeutenden Teil ausschliesslich auf die Impfung zurück.

Der Heymanssche Impfst of $f$ wird nach den Mitteilungen über ca. 20000 Impfungen von den Tieren gut vertragen, letzteres gilt auch von ca. 30000 Impfungen mit dem Antiphymatol Ḱlimmers (Engdahl und Glöckner, Tierärztliche Rundschau 1908, Klim mer, Zeitschrift für Tiermedizin 1908, S. 123, Glöckner, Berl. Tierärztliche Wochenschr. 1908 etc.).

\section{Die Wirksamkeit der verschiedenen Impfverfahren.}

Das endgiltige Urteil über die Leistungsfähigkeit einer für die Praxis bestimmten Impfmethode kann man natürlich auch nur a u $\mathrm{f}$ Grund der Erfahrungen in der Praxis fällen. Da aber das Sammeln einer hinlänglichen Anzahl von Beobachtungen aus der Praxis und ganz besonders hier bei der Tuberkulose sehr lange Zeit erfordert, so hat man vielfach lediglich zur Abkürzung der Zeit versucht, auf 
dem Wege der künstlichen oder verstärkten natürlichen Infektion sich frühzeitiger einen Einblick in die Wirkung der Impfung zu rerschaffen. Die bei diesen Infektionsversuchen gewonnenen Ergebnisse vermögen zwar bei den Impfverfahren, bei denen zur Bekämpfung der Tuberkulose ausschliesslich der durch die Impfung erzielte Impfschutz benutzt wird, einen erheblichen Einblick in die Leistungsfähigkeit der betreffenden Verfahren zu gewähren, dagegen können diese Infektionsversuche von der Wirksamkeit derjenigen Tuberkulosetilgungsverfahren, bei denen die Impfung durch eine Reihe hygienischer Massnahmen unterstützt wird, sehr leicht ein falsches Bild entwerfen. Ja sie sind bei diesen von hygienischen Massnahmen unterstuitzten Verfahren insofern paradox, als der innerhalb mässiger Grenzen vorgeschriebene Schutz vor einer Infektion gerade in das Gegenteil, die künstliche oder verstärkte natürliche Infektion, umgewandelt wird. Es ist hinsichtlich der künstlichen Infektionsversuche auch daran zu erinnern, dass die künstliche Infektion in der Regel unvergleichlich stärker ist, als die natürliche zu sein pflegt, sich aber andererseits von dieser durch ihre zeitliche Beschränkung unterscheidet.

\section{a) Ergebnisse des künstlichen und verstärkten natiirlichen Infek- tionsversuches.}

Wenn die Prüfung des gegen die Tuberkulose zu erzielenden nur relativen Schutzes überhaupt brauchbare Ergebnisse liefern soll, so muss die künstliche Infektion so klein sein, dass durch sie bei den nicht schutzgeimpften Kontrolltieren nur gerade noch die gewünschte krank machende Wirkung eintritt. Werden dagegen zu grosse Dosen gewählt, so rermögen dieselben selbst die geimpften Tiere nicht zu bewältigen, und das Bild der Schutzwirkung der Impfung wird verschwommen und verzert. Weiterhin ist $z u$ fordern, dass nur solche Tiere zu diesem Versuche benutztwerden, die zu Beginn der Versuche frei von Tuberkulose sind.

Wenden wir uns den Ergebnissen der künstlichen und verstärkten natürlichen Infektionsversuche zu, so ist vorauszuschicken, dass solche bei allen vier Verfahren vorliegen. Da die künstlichen Infektionsversuche mit Rindertuberkelbazillen in verschiedener Menge und auf verschiedenen, wenn auch vorwiegend auf intravenösem Infektionsweg ausgeführt worden sind, so ist es unmöglich, die Ergebnisse dieser. Versuche miteinander direkt zu vergleichen.

Bei dem beschränkten Wert, welcher den künstlichen und verstärkten natürlichen Infektionsversuche für die Beurteilung der verschiedenen, zum Teil gleichzeitig von hygienischen Massnahmen unterstützten Tuberkuloseimpfverfahren zukommt will ich mich begnügen, die wichtigsten Ergebnisse in nachfolgender Tabelle wiederzugeben: 
Tabelle 1.

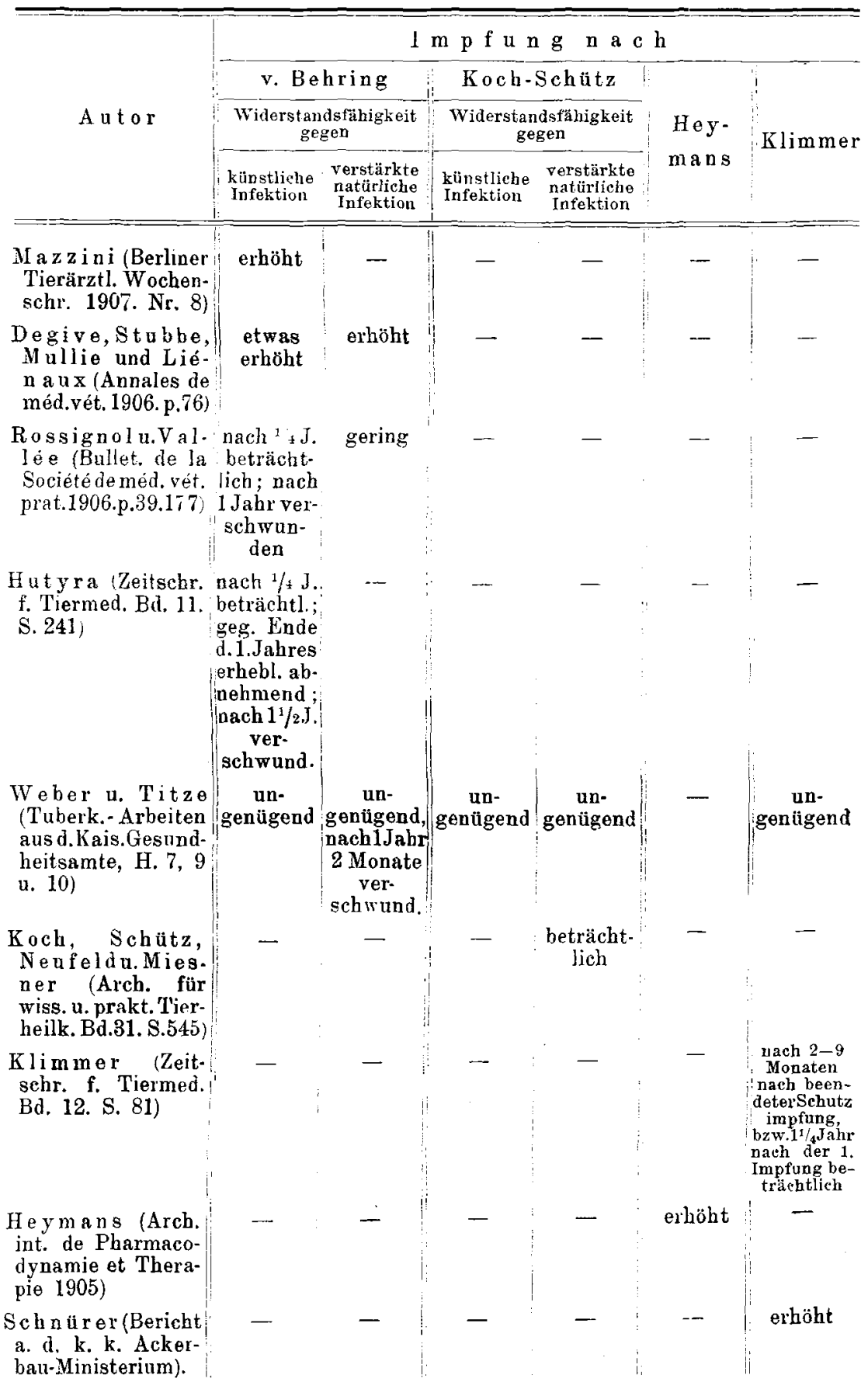


Aus der Zusammenstellung ist ersichtlich, dass die Ergebnisse der Schutzpröfungen ein- und desselben Verfahrens nicht unerheblich von einander abweichen. Während die meisten Forscher eine erhebliche Schutzwirkung des v. Beh. ringschen Verfahrens im ersten Jahre nach beendeter Impfung nachweisen konnten, wurde bei den Versuchen von Weber und Titze eine ungenügende Widerstandsfähigkeit geimpfter Tiere gegen die Tuberkulose beobachtet. Ebenso ungünstig fiel die Prüfung der letzten beiden Autoren hinsichtlich der Schutzwirkung des Taurumans und Antiphymatols aus, welche nach den Versuchen von Koch, Schütz, Neufeld und Miessner, bezw. Klimmer und Schnürer, eine beträchtliche Widerstandsfähigkeit verleihen. Dass bei diesen Infektionsversuchen sehr leicht solche abweichende Ergebnisse erhalt $\_$n werden können, und worin der Grusd hierfür zu suchen ist, habe ich schon erwähnt. Wurden die Versuchstiere erst etwa ein Jahr oder später mach beendeter Schutzimpfung zu dem Infektionsversuch benutzt, so wurde selbst von den Aatoren, die bei früher durchgeführten Infektionen günstige Ergebnisse beobachtet batten, nunmehr ein ungenügender Schutz gefunden. Aus diesen Versuchen schliesse ich:

1. Durch die Torbehandlung mit verschiedenen Tuberkuloseimpfstoffen kann man den Rinclern leicht einen beträchtiichen Schutz vor einer Tuberkuloseinfektion verleihen.

2. Die Schutzwirkung der Tuberkuloseimpfrerfahren ist von beschränkter Dauer: sie beträgt nur etwa ein Jahr.

Da ein einjähriger Schutz bei der Tuberkulose praktisch ron nur sehr beschränktem Wert ist, so muss die Schutzwirkung durch alljährliche Nachimpfung verlängert werden und damit die Impfung erst einen praktischen Wert als Bekämpfungsmittel gegen die Tuberkulose erhalten. In Hinblick darauf, dass altere Tiere Aufschwemmungen von rirulenten Tuberkelbazillen vielfach nicht gut vertragen (v. Behring) und vor allem aus den auf Seite 6 u. ff. erwähnten sanitären Gründen können die Milchtiere, und das sind die überwiegende Mehrzahl der älteren Rinder, nur einew für Menschen sicher unschädlichen Impfrerfahren unterzogen werden. Nach den früheren Darlegungen kann in dieser Richtung nur die Impfung mit einem nichtinfektiösen 'Tuberkuloseimpfstoff (Antiphymatol) in Frage kommen.

\section{Ergebnisse der Praxis.}

Um die Leistungsfähigkeit der einzelnen Tuberkuloseimpfverfahren in der Praxis zu beurteilen, hat man sich zum Teil auf die Ergebnisse der Tuberkulinprobe gestützt. Dass die hierbei gewonnenen Resultate einen geringeren wissenschaftlichen Wert besitzen als die Befunde einer genauen pathologisch-anatomischen Cntersuchung dürfte wohl keinem Zweifel unterliegen. Die Tuberkulinprobe ist als Prüfstein für eine erfolgreiche Impfung mit dem Bovovakzin, welche zunächst ausschliesslich berücksichtigt wird, mehrfach benutzt werden, so von v. Behring und seinem Yitarbeiter Römer, ferner von Hutyra, Eber, Ebeling, Schricker, Klimmer, Lorenz, 
Régner und Stenström etc. Die genannten Autoren meinen, dass die Tuberkulinprobe dann brauchbare R€sultate liefert, wenn seit der letzten intravenösen Injektion des Bovovakzins etwa ein Jahr verflossen ist. Einen Überblick über die bei den mit Bovovakzin geimpften Rindern erhaltenen Ergebnisse geben die beiden nachfolgenden Tabellen 2 und 3 .

Tabelle 2 .

\begin{tabular}{|c|c|c|}
\hline A utor & $\begin{array}{c}\text { Von den nach v. B ehring } \\
\text { schutzgeimpften Rindern } \\
\text { reagierten bei der später } \\
\text { vorgenommenen Tuber- } \\
\text { kulinprobe }\end{array}$ & $B$ e mel $k u n g$ \\
\hline $\begin{array}{l}\text { Ebeling (zitiert nach } \\
\text { Römer, Bericht iuberd. } \\
\text { VII. Int. Tierärztl. Kon- } \\
\text { gress 1905. S. } 83 \text { ) }\end{array}$ & $28 \%$ & 2-3 jährige Impflinge \\
\hline $\begin{array}{l}\text { Klimmer (Bericht über } \\
\text { das Veterinärwesen im } \\
\text { Königr. Sachsen für das } \\
\text { Jahr 1903. S. 321) }\end{array}$ & $30 \%$ & - \\
\hline $\begin{array}{l}\text { Schricker (Wochenschr. } \\
\text { f. Tierbeilk. a. Viehzucht } \\
\text { 1906. Nr. 7) }\end{array}$ & $50 \%$ & - \\
\hline \multirow[t]{2}{*}{$\begin{array}{l}\text { Eber (Zentralbl. f. Bad. } \\
\text { etc. Orig.-Bd. } 44 . \text { H. } 5 \\
\text { u. 6) }\end{array}$} & $45,7 \%$ & $\begin{array}{l}\text { von } 81 \text { mindestens } 1 \text { Jahr } \\
\text { nach der Impfung ge- } \\
\text { prüften Rindern }\end{array}$ \\
\hline & $38,8 \%$ & $\begin{array}{l}\text { von } 18 \text { desgl, vor der } \\
\text { ersten Impfung auf Tu- } \\
\text { berkulin nicht reagieren- } \\
\text { den Rindern }\end{array}$ \\
\hline $\begin{array}{l}\text { D a m m an (XXXV. Plenar- } \\
\text { versammlung d. Deutsch. } \\
\text { Landwirtschaftsrat) }\end{array}$ & $61 \%$ & - \\
\hline $\begin{array}{c}\text { A ndracek (Tierärztliches } \\
\text { Zentralbl. 1907. Nr. 11) }\end{array}$ & - & - \\
\hline $\begin{array}{l}\text { Loren z (Zeitschr. f. Tier- } \\
\text { med. Bd. 9. S. 131) }\end{array}$ & $34,5 \%$ & - \\
\hline $\begin{array}{l}\text { v. Behring (Beiträge zur } \\
\text { exper. Therapie. H. 10) }\end{array}$ & $43,6^{\circ}, 0$ & - \\
\hline
\end{tabular}

Die bei vorstehenden Tuberkulinproben erhaltenen Reaktionsprozente schwanken zwischen 28 und 61 ; würde ich sie für einzelne Bestände getrennt angegeben haben, so würden zum Teil noch wesentlich höhere Werte erhalten worden sein. Schon aus dieser Zusammenstellung ist zu entnehmen, dass die v. Behrings che Bovovakzination keineswegs den praktischen Bedürfnissenentspricht. Wie ungenügend die Schutzwirkung der Bovovakzination ist, geht 
noch deutlicher aus nachfolgender Zusammenstellung hervor, in die zum Vergleich die Reaktionsprozente der nicht geimpften Kontrolltiere der betr. Bestände mit aufgenommen worden sind.

Tabelle $\hat{0}$.

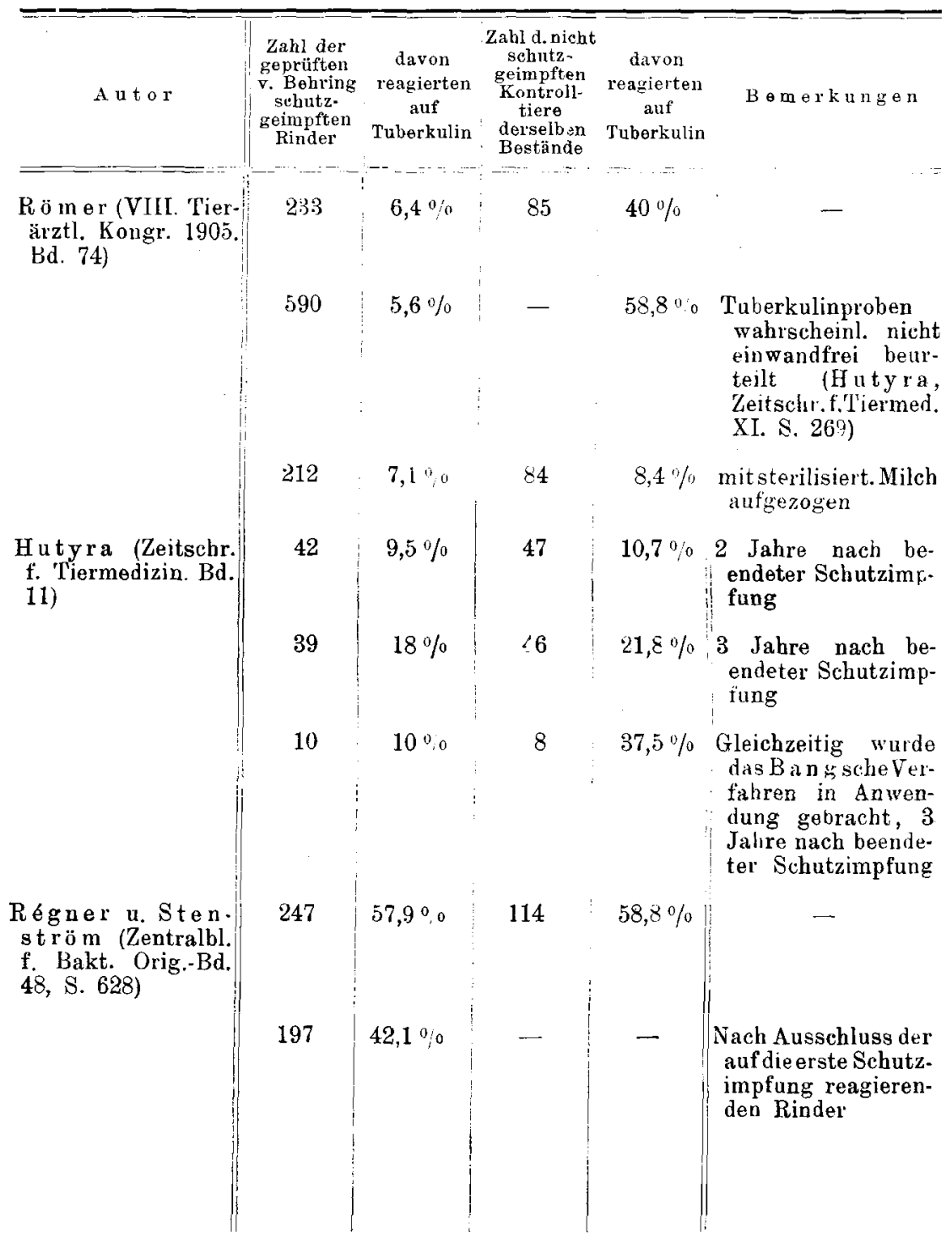

Von den in die Tabelle 3 aufgenommenen Ergebnissen können nur die beiden ersten Angaben $\mathrm{Römers}$ befriedigen. Da aber die Tuberkulinproben wahrscheinlich nicht einwandfrei beurteilt, vielleicht. 
sogar nicht einmal richtig durchgeführt worden sind, wie dies $\mathrm{Hu}$. tyra auch nach meinem Dafürhalten zutreffend begründet hat, ausserdem mit allen anderen Angaben in auffallendem Kontrast stehen, möchte ich denselben keine Bedeutung beilegen. Alle anderen Ergebnisse höchstens noch mit Ausnahme der kleinen uritten Versuchsreihe Hutyras sind vollkommen unbefriedigend und erhärten das an die Zusammenstellung 2 geknüpfte Urteil über die Bovovikzination v. Behrings.

C̈ber die der Bovorakzination unterworlenen Rinder liegen bereits mehrere Schlachtbefunde vor. Ich stelle sie aus Platzmangel in nachfolgender Tabelle 4 zusammen und bin mir dabei sehr wohl bewusst, dass dabei eine Reihe wertvoller Details leider verloren gehen.

Tabeile 4.

\begin{tabular}{|c|c|c|c|}
\hline $\begin{array}{c}\text { A utor } \\
\text { und } \\
\text { Literaturangabe }\end{array}$ & $\begin{array}{c}\text { Zahl der } \\
\text { geschlachteten } \\
\text { bovovakzinierten } \\
\text { Rinder }\end{array}$ & $\begin{array}{c}\text { davon } \\
\text { tuberkulös }\end{array}$ & Bemerkungen \\
\hline $\begin{array}{l}\text { Ebeling nach Römer } \\
\text { (Tierärztl. Kongr. } 1905 \text {. } \\
\text { 3. Bd. S. 74) }\end{array}$ & 37 & $16,5 \%$ & - \\
\hline $\begin{array}{l}\text { Strelinger (Zeitschr. f. } \\
\text { Tiermedizin. } 118 \text { ) }\end{array}$ & 5 & $40 \%$ & - \\
\hline $\begin{array}{l}\text { Sch ricker (Wochenschr, } \\
\text { f. Tierheilk. w. Viehzucht } \\
\text { 1906. Nr. 7) }\end{array}$ & 3 & $33,3 \%_{/ 0}$ & - \\
\hline $\begin{array}{l}\text { Kö m e r (Tierärztl. Kongr. } \\
\text { 1905. Bd. 3. S. 74) }\end{array}$ & 109 & $16,5 \%$ & $\begin{array}{l}\text { umfisst vermutlich } \\
\text { einen Teil der an. } \\
\text { deren Angaben }\end{array}$ \\
\hline \multirow{2}{*}{$\begin{array}{l}\text { Eber (Zentralbl. f. Bakt. } \\
\text { Bd. 44. H. 5/6) }\end{array}$} & 19 & $47,4 \%$ & - \\
\hline & 13 & $23,10^{\circ}, 0$ & $\begin{array}{l}\text { nach Abzug der mög- } \\
\text { licherweise vor der } \\
\text { 1. Impfung bereits } \\
\text { tuberkulös erkrank- } \\
\text { ten Tiere }\end{array}$ \\
\hline $\begin{array}{l}\text { Régner u. Stenström } \\
\text { (Zentralbl. f. Bakt. Bd. } \\
\quad \text { 48. S. 628) }\end{array}$ & 13 & $46,1 \%$ & $"$ \\
\hline $\begin{array}{l}\text { L o renz (Tierärztl. Kongr. } \\
\text { Bd. 3. S. 503) }\end{array}$ & 21 & $19,5 \%$ & - \\
\hline
\end{tabular}

Wie aus obiger Zusammenstellung ersichtlich ist, sprechen auch die Ergebnisse der Obduktionen, wie 
schon zuvor die der Tuberkulinproben, welche an den nach v. Behring bovovakzinierten Rindern gewonnen worden sind, nicht dafür, dass mit dem Tuberkuloseschutzimpfungsverfahren nach r. Behring (Bovovakzination) die Tuberkulose der Rinder erfolgreich bekämpft werden kann.

Dieses recht ungünstige Urteil muss überraschen, wenn man damit lediglich die Ergebnisse vergleicht, welche bei den im ersten Jahre nach beendeter Schutzimpfung vorgenommenen künstlichen Infektionsversuchen gewonnen wurden. Während bei diesen künstlichen Infektionsversuchen, wie schon hervorgehoben, mit Ausnahme jener von Weber und Titze, bei denen die Infektion wohl zu kräftig gewählt sein dürfte, eine erhöhte, zu einem grossen Teil sogar beträchtlich erhöhte Widerstandsfähigkeit festgestellt wurde, war eine solche bei den Versuchen in der Praxis fast vollkommen zu vermissen. Die Antwort aut dieses zunächst sonderbar erscheinende Verhalten geben, wie ebenfalls schon betont, die in späterer Zeit nach beendeter. Schutzimpfung vorgenommenen künstlichen Infektionsversuche. Sie zeigen; dass der Impfschutz schon nach einem Jahr verschwindet. Nach dieser Zeit sind die Impflinge nicht mehr geschützt, sie fallen dann der Tuberkuloseinfektion anheim. Da eine Nachimpfung im späteren Alter mit dem virulente Tuberkelbazillen enthaltenden Bovovakzin nicht angängig ist, so muss infolgedessen die Bovovakzination nach v. Behring als Bekämpfungsmittel der Rindertuberkulose in der Praxis versagen.

In ganz gleicher Weise liegen auch die Verhältnisse bei der Impfung mit dem Tauruman nach Koch-Schütz, ron dem Hutyra Zeitschrift f. Tiermedizin Band 11, Seite 24) nachgewiesen hat, dass die einmalige Implung etwa den gleichen Schutz gewährt wie die zweimalige Bovovakzination.

Aus der Praxis liegen meines Wissens keine Ergebnisse von Tuberkulinproben oder Obduktionen vor. Auf dem IX. Internat. Tierärztl. Kongress erklärte Miessner, welcher das Impfverfahren mit Tauruman gemeinschaftlich mit Koch, Schütz und Neufeld ausgearbeitet hat, dass die Taurumanimpfung zur Tuberkulosebekämpfung nicht geeignet sei.

In neuerer Zeit teilten Weber und Titze mit, dass $\mathrm{durch}$ Bovovakzin- und Taurumanimpfungen Augen- und Gelenktuberkulose, verursacht durch die Bovorakzin. und Taurumantuberkelbazillen, zuweilen hervorgerufen wa rden. 
Über die Leistungsfähigkeit des Heymansschen Verfahrens hat meines Wissens bisher nur Heymans selbst berichtet. Seinen Mitteilungen in Archives internationales de Pharmacodynamie et de Therapie 1908, Vol. XVIII p. 179 entnehme ich folgende Angaben.

Vorauszuschicken ist, dass Heymans zurzeit sowohl auf Tuberkulin nicht reagierende als auch reagierende Tiere impft, um erstere zu schützen, letztere zu heilen. Wenden wir uns zunächst seinen Angaben über die Schutzwirkung seines Impfstoffes zu, so ist folgender Versuch zu erwähnen: 9 geimpfte und 9 nicht geimpfte, auf Tuberkulin nicht reagierende Rinder wurden zusammen $3-17$ Monate lang der gleichen natürlichen Ansteckung in verseuchten Beständen ausgesetzt. Mit Ausnahme von 1 geimpften und 1 Kontrolltier, von denen Heymans das erstere als nicht tuberkulös, das andere als tuberkulös hält, sind alle Versuchstiere geschlachtet worden. Sektionsbefund: Von den 8 geschlachteten geimpften lieren zeigte 1 "des lésions manifestement tuberculeuses" und 1 "des lésions suspectes", die übrigen 6 Tiere $(=75 \%)$ waren frei von Tuberkulose. Von 8 geschlachteten Kontrolltieren waren $3\left(=37, \tilde{0}^{\circ} \%\right)$ frei ron Tuberkulose; die übrigen 5 tuberkulös; eines darunter in so hohem Grade, dass das Fleisch für ungeeignet zum Genusse für Menschen erklärt werden musste.

Die weiteren Angaben über Sektionsbefunde lauten:

Seit Dezember 1905 bezw. Januar 1906 wurden 2 Bestände mit etwa $1<8$ resp. 40 Rindern der Impfung unterzogen. Vom Mai bis Dezember 1906 sind 45 Tiere seziert worden, von denen nur 2 frei von Tuberkulose waren. Von 68 im Januar 1907 sezierten Tieren waren 17 frei von Tuberkulose, die anderen zeigten „des lésions tuberculeuses fréquemment en régression manifeste".

Insgesamt sind bis zum 31. Dezember 1906 über 400 Sektionen ausgeführt worden. Darunter befinden sich mehr als 100 Tiere, welche sowohl auf die erste, wohl kurz vor die Schutzimpfung fallende, 1906 vorgenommene, als auch auf eine zweite im Jahre 1907 durchgeführte Tuberkulinprobe nicht reagiert haben. (Wie nachfolgend zu erwähnen ist, zeigten andere ebenfalls auf die erste Probe nicht reagierende, schutzgeimpfte Tiere bei der zweiten Probe eine positive Reaktion). Von diesen ersteren wiesen bei der Schlachtung 5 oder 6 "makroskopische" Tuberkulose auf.

Weiterhin teilt $\mathrm{Heymans}$ eine grosse Anzahl von Tuberkulinproben an geimpften Tieren mit. Letztere reagierten zu einem erheblichen Teil vor der Impfung. Auch diese wurden der Impfung und im weiteren Verlauf auch weiteren Tuberkulinproben unterzogen. Die Versuchstiere, welche a uf die erste, wohl vor der Schutz- 
impfung ausgeführte Tuberkulinprobe nicht reagiert haben, wurden der Schutzimpfung wiederholt unterzogen und in der Zwischenzeit mit Tuberkulin nachgeprüft. Hierbei wurde gefunden, dass von 15 Tieren der ersten Versuchsreihe 3 Stïck nach 2 Jahren und 12 Stück nach $1 \mathrm{Jahr}$ auf Tuberkulin nicht reagierten. Weiterhin berichtet Heymans über 509 schutzgeimpfte Rinder, welche auf die erste Tuberkulinprobe nicht reagierten. $1 \mathrm{Jahr}$ später wurden sie von neuem mit Tuberkulin geprüft. es reagierten nunmehr $4 \%$ z weifelhaft und $20 \%$ positir. Diesen schutzgeimpften Tieren stehen 505 nicht schutzgeimpfter Tiere gegenüber. Sie sind zum kleineren Teile zugekauit, zum grösseren Teil in denselben Beständen seit der 1. Tuberkulinprobe geboren und ca. ${ }^{1}$, Jahr in denselben Stallungen gehalten worden. Bei der Tuberkulinprobe dieser bis zu einem gewissen Grate als Kontrollen anzusehende, jedoch ror einer Milchinfektion nicht geschützten Tiere reagierten $7 \%$ zweifelhaft und ebenfills $20 \%$ positiv.

Heymans zieht hieraus den Schluss, dass die gesunden Impflinge sich weniger infizieren als die nicht geimpften. Hält man sich lediglich an die nackten Zahlen und lässt alles andere unberücksichtigt, so würde sich ergeben, dass in der Zeiteinhejt ron den nichtgeimpften Tierenetwa diedoppelte Anzahlals von den geimpften der Tuberkuloseinfektion anheimfallen.

Hinsichtlich der Heilerfolge teilt $\mathrm{H}$ eymans ausschliesslich nur das summarische Ergebnis von Tuberkulinproben an anfangs reagierenden, hierauf geimpften und sodann wieder mit Tuberkulin geprüften Rindern mit. Das schliessliche Ausbleiben einer Tuberkulinreaktion an Tieren, welche z. T. wiederholt der Tuberkulinprobe und der Impfung mit Tuberkulosepräparaten unterworfen worden sind, kann noch nicht als ein Beweis für eingetretene Heilung angesprochen werden.

Es bleibt nunmehr nur noch übrig, auf die in der Praxis gesammeiten Ergebnisse des Klimmerschen Tuberkulosetilgungsverfahrens mit $\mathrm{Hilfe}$ von Antiphymatol einzugehen.

Früher, als ich die Impfungen noch intravenös ausfülırte, habe ich die Schutzwirkung durch Tuberkulinproben zu kontrollieren versucht. Hierbei fand ich (Bericht über das Veterinärwesen im Königreich Sachsen auf das Jahr 1905,, Seite 368), dass von etwa 200 intravenös schutzgeimpften Kälbern, die vor der Immunisierung mit Tuberkulin vorgeprüft und frei befunden waren und bei denen die Schutzimpfung zum Teil (60) über ein Jahr zurücklag, kein einziges reagierte, dagegen zeigten von 15 mit ersteren völlig gleichgehaltenen Kontrolltieren $6-40 \%$ eine positive Reaktion. Die gleichen Ergebnisse mit meinem 
Impistoff hat auch Engdahl-Lautakyla ${ }^{1}$ ) hinsichtlich schutzgeimpfter Tiere erhalten. Über Kontrolltiere erwähnt er nichts.

Später, als ich ausschliesslich zur sublutanen Impfmethode überging und die alljährlichen Nachimpfungen einführte, habe ich die Prüfungen mit dem Tuberkulin aufgeben müssen, da die nichtinfektiösen Impfstoffe (ganz besonders die bei eigenen Versuchen viel benutzten abgeschwächten Menschentuberkelbazillen) nach subkutaner Applikation selır häufig eine jahrelange Tuberkulinüberempfindlichkeit zurücklassen. Von da ab war ich zur Beurteilung meiner Nethode ausschliesslich auf die Obduktionsbefunde angewiesen.

Von den Tieren, die auf die vor der Schutzimpfung vorgenommene Tuberkulinprobe nicht reagiert haben, sind bisher 68 Stück pathologisch-«natomisch untersucht worden.

Die erste Schutzimpfung wurde von diesen Rindern überlebt ion:

\begin{tabular}{|c|c|c|c|c|}
\hline 14 & Stück & 1 & -3 & Monate \\
\hline 7 & , & 3 & -6 & 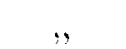 \\
\hline 8 & ,: & 6 & -9 & : \\
\hline 3 & ., & 9 & -12 & \\
\hline 10 & , & 1. & -1 & 2 Jahre. \\
\hline 9 & $"$ & $1^{1}$ & $2-2$ & "4 \\
\hline 11 & $\because$ & 2 & & , \\
\hline 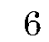 & $\because$ & 3 & -4 & ,. \\
\hline
\end{tabular}

Von diesen 68 schutzgeimpften Rindern wies nur eins tuberkulöse Veränderungen in der Lunge und eins in den Bronchial- und Mediastinaldrüsen auf; alle anderen 66 schutzgeimpften Rinder waren frei von Tuberkulose. Jene zwei erwähnten schutzgeimpiten Rinder, welche geringlügige tuberkulöse Prozesse erkennen liessen, waren, wie ein sehr grosser Teil der frei befundenen Rinder, in Beständen untergebracht, in denen entgegen der Vorschrift keinerlei hygienische Massnahmen durchgeführt wurden. Ausserdem ist bezüglich dieser zwei Impflinge noch zu erwähnen, dass die der ersten Schutzimpfung vorausgeschickte thermische Tuberkulinprobe insofern nicht hinlänglich sachgemäss durchgeführt worden ist, als die Temperaturen nach der Tuberkulininjektion nicht genügend aufgenommen worden sind. Bei dem einen Tier ist nur von der 9.-19., bei dem anderen von der 9,-15. Stunde nach der Einspritzung zweistündlich, hierauf bei letzterem noch einmal zur 19. und 23. Stunde die Temperatur aufgenommen worden.

In manchen Beständen waren die Kontrollrinder stark verseucht, in einem sogar ausnahmslos tuberkulös.

1) Brief liche Mitteilung. 
Diese Ergebnisse sind zum Teil ron mir und meinen Mitarbeitern Kiessig und Assmann, zum Teil von Herrn Geheimrat JohneKleinsedlitz, zum Teil ron praktischen Tierärzten, die mit meinen Impfstoffen gearbeitet haben, erhoben worden (Deutsche Tierärztliche Wochenschrift 1909, Nr. 1 und Tierärztliche Rundschau 1908 S. 419). Während ich die Impflinge nach Möglichkeit vor einer Milchinfektion schützen (Milch von nicht reagierenden Ammenkühen oder pasteurisierte Milch, oder wenigstens Milch von nur einer Kuh und nach Möglichkeit keine Mischmilch), die vor der Impfung nicht reagierenden Tiere in geschlossener Reihe aufstellen und, wenn sie in doppelten Reihen mit den Köpfen gegenüberstehen, auch nicht reagierende Tiere gegenüber anbinden und als tuberkulös erkannte Tiere (auf Eutertuberkulose wird aus sanitären Gründen hin untersucht), abschlachten lasse, haben verschiedene Impftierärzte diese Hilfsmittel nicht angewandt und sich ausschliesslich a uf die Impfung beschränkt. Trotzdem sie bisher die gleichgünstigen Ergebnisse erzielt haben, möchte ich die Lnterstützung der Impfung durch die erwähnten zumeist leicht durchführbaren Massnahmen, wie sie zum Teil in den Bangschen und Ostertagschen Verfahren enthalten sind, zur Erzielung eines möglichst sicheren Erfolges nicht fallen lassen.

In neuerer Zeit findet der Impfstoff auch bei bereits tuberkulös infizierten Rindern Anwendung. Es hatte sich nämlich gezeigt, dass einerseits ein Aufblühen schlummernder Tuberkulose nicht zu befürchten ist und andererseits der vorhandene tuberkulöse Prozess zum Stillstand kommt, ohne dass frische tuberkulöse Prozesse auftreten, wenn die Tuberkulose noch nicht $\mathrm{zu}$ weit vorgeschritten ist und es sich um noch kräftige, reaktionsfähige Tiere handelt.

Von den auf Tuberkulin reagierenden, also als tuberkulös anzusprechenden Rindern sind bisher 59 zur Untersuchung im ausgeschlachteten Zustand gekommen. Von diesen 59 Rindern erwiesen sich 24 bei der Fleischbeschau als frei von Tuberkulose. Die erste Impfung lag

zurück.

$\begin{array}{crccc}\text { bei } & 3 & \text { Tieren } & 2-3 & \text { Monate, } \\ \text {, } & 3 & , & 3-6 & , \\ \text {, } & 12 & , & 6-9 & , \\ \text {, } & 5 & \text {, } & 9-12 & ., \\ , & 1 & , & \text { über } 1 \text { Jahr }\end{array}$

Bei diesen 24 vor der ersten Heilimpfung auf 'Tuberkulin reagierenden, somit als tuberkulös anzusprechenden Tieren dürfte wohl 
sicherlich der tuberkulöse Prozess nach der Impfung keine Fortschritte gemacht haben, sonst müsste er wohl bei der Obduktion gefunden worden sein. Es ist vielmehr anzunehmen, dass die tuberkulöse Erkrankung zum Stillstand, in frischen, jungen Prozessen wohl auch zur vollständigen Rückbildung gekommen ist.

Bei weiteren 6 der Heilimpfung unterzogenen Tieren wurden durch die Obduktion neben älteren auch frische Veränderungen festgestellt. Die erste Impfung lag

$$
\begin{aligned}
& \text { bei } 1 \text { Tier } 3 \text { Monate, } \\
& \text { "2 Tieren } 10 \text { " } \\
& \text { "1 Tier } 1 \text { Jahr }
\end{aligned}
$$

zurück und bei 2 Tieren waren keine Angaben gemacht worden, wohl aber bemerkt der betreffende Tierarzt, dass in den beiden letzten Fällen die Tuberkulose ,allerdings schon weit vorgeschritten", also zur Impfung nicht mehr geeignet war. Ausserdem erfolgte die Schlachtung schon kurze Zeit nach der Impfung, so dass gar keine Zeit zur Verheilung (Abkapselung) war. Die beiden 10 Monate nach der ersten Impfung geschlachteten Rinder waren nur ein- bezw. zweimal entgegen der Vorschrift, welche für die betreffende Zeit eine dreimalige Impfung anweist, behandelt worden.

Die übrig bleibenden 29 tuberkulösen heilgeimpften Rinder liessen bei der Obduktion eine deutliche Abkapselung (Verheilung) der bestandenen tuberkulösen Veränderungen erkennen; frische Prozesse fehlten vollkommen. Verschiedene Tierärzte (Seeliger, Hauptmann, Jüterbock usw). heben in ihren Berichten mehrfach hervor, dass sie eine so starke abgekapselte Tuberkulose bei nicht geimpften Tieren bisher niemals beobachtet haben, was ich nach meinen eigenen Erfahrungen nur bestätigen kann. Unter anderen schreibt Glöckner (Tierärztl. Rundschau 1908, S. 419 bezw. Berl. tierärztl. Wochenschr. 1909, S. ¿92) hierüber:

„Bemerkt sei noch, dass die genannten schutz- und heilgeimpften Tiere in notorisch verseuchten Ställen die ganze Zeit gestanden haben und ich derart abgeheilte Form der Tuberkulose bislang nicht an Schlachtstiucken aus jenen Ställen beobachtet habe...." „Ich war erfreut über diese Befunde, da ich aus fraglichen Ställen bei nichtgeimpften Schlachttieren immer eine Tuberkuloseform fand, welcher ein besonderer heftiger Virulenzgrad inne zu wohnen schien (Ausbreitung und wenig Neigung zur Abkapselung und Verkalkung)."

Drei weitere Tierärzte erwähnen:

„Die schutzgeimpften Rinder erwiesen sich bei der Schlachtung frei von Tuberkulose; bei den beilgeimpften trat trotz der zum Teil 
schweren Erkrankung Heilung mit aussergewöhnlich starker Abkapselung ein." (Illustrierte Landwirtschaftliche Zeitung 1910, Seite 171.)

Die erste Impfung lag bei diesen 29 Rinder, bei denen die Tuberkulose durch die Impfung zur Heilung kam,

zurück.

$$
\begin{aligned}
& \text { bei } 2 \text { Rindern } 2-3 \text { Monate, } \\
& \text {, } 10 \quad, \quad 3-6 \quad \text {.. } \\
& , \quad 5 \quad, \quad 6-9 \quad, \\
& \begin{array}{lllll}
. . & 4 & . & 9-12 \quad . .
\end{array} \\
& \text { " } 15 \quad \text {, } \quad 5 \quad 1-1 \frac{1}{2} \text { Jahre } \\
& \text {., } 3 \quad . . \quad \text { über } 1^{1 / 2} \text { Jahre }
\end{aligned}
$$

Von 99 heilgeimpiten tuberkulösen Rindern ist also bei 13 der Prozess zum Stillstand, zur Heilung, gekommen. Gewiss kann ein tuberkulöser Prozess auch ohne Impfung abheilen und abkapseln; aber es ist dies loch nicht die liegel, lie wir jedoch nach der Impfung, wie gezeigt, bisher bestätigt gefunden haben.

Über diejenigen 'liere, die bisher nicht geschlachtet worden sind, und die vor der Impfung Symptome der Tuberkulose (Husten, rauhes Haar, trüben Blick, schlechten Ernährungszustaud) zeigten, wird von verschiedenen Tierärzten (Hein rich, Sch rader) mitgeteilt, dass die erwähnten mehr oder weniger für Tuberkulose sprechenden Erscheinungen (namentlich bei gleichzeitig positiver Tuberkulinprobe) in einigen Wochen nach den Impfungen verschwanden.

Andere Tierärzte heben herror, dass ,der Nährzustand und der Milchertrag tuberkulöser Rinder sich nach der Impfung hob, so dass sich schon hierdurch die Kosten der Impfung in kurzer Zeit bezahlt machen. (Illustrierte Landwirtschaftliche Zeitung 1910 , Seite 171.)

Die Frage der Heilung bezw. des Stillstandes der Tuberkulose ist ganz wesentlich schwieriger zu beurteilen als die Schutzwirkung und erfordert zu ihrer endgültigen Beantwortung natürlich ein sehr grosses Material. Es wird die Aufgabe der folgenden Jahre sein, dieses zu sammeln.

Nach dem bisher vorliegenden, vorstehend mitgeteilten Beobachtungsmaterial über die Wirksamkeit des Klimmerschen Tuberkulosetilgungsverfahrens mit Hilfe von Antiphymatol hat genanntes Verfahren den Anforderungen der Praxis entsprochen. 


\section{Zusammenfassung.}

1. In der Praxis sind z. Z. rier Impfrerfahren gegen die Tuberkulose eingeführt worden. Es sind lies die Bovovakzination v. Behrings, die Impfung mit dem l'auruman na:h KochSch ït $z$, die Heymanssche Schutz- und Heilimptung mit in Schilfsäckchen eingeschloseenen Tuberkelbazillen und das Klimmer. sche Tuberkulosetilgungsverfalıren mit Hilfe von Antiphymatol.

2. Bei der Beurteilung obiger Impfrerfahren sind folgende Punkte zu berücksichtigen:

1. Thre Ungefährlichkeit für Menschen,

a) beim Impfakt,

b) bei der Wartung der Impflinge,

c) beim Genuss von Fleisch und Milch der Impflinge,

2. ihre Ungefährlichkeit für die Impflinge,

3. ihre Wirksamkeit.

3. Für die Gefährlichkeit der Impfrerfahren für Menschen ist die Virulenz der als Impfstoff verwendeten Menschentuberkelbazillen ron ausschlaggebender Bedentung. Während das Bovovakzin v. Behrings, das Tauruman Koch-Schütz und der He y m a n sche Impfstoff virulente Tuberkelbazillen enthalten, kommen virulente Tuberkelbazillen in dem Antiphymatol Klimmers nicht vor und treten auch nach einfachen Tierpassagen nicht auf.

Hinsichtlich des Heymansschen Impfstoffes ist noch hervorzuheben, dass die Tuberkelbazillen in Schilfsäckchen eingeschlossen sind. Solange die Säckchen vollkommen dicht sind, ist auch dieser Impfstoff für Menschen ungefährlich, er wird aber gegebenen Falles gefährlich werden können, wenn die Säckchen zerreissen.

4. Die Tuberkuloseimpfstoffe, welche virulente Tuberkelbazillen enthalten, können beim Impfakt gewisse Gefahren für die menschliche Gesundheit bieten, namentlich gilt dies von dem Tauruman und dem Bovovakzin. Der Heymans sche Impfstoff kann, da hier die Tuberkelbazillen in Schilfsäcken und diese wiederum in Gelatinekapseln eingeschlossen sind, hinsichtlich des Impfaktes wohl als ungefäbrlich angesehen werden, sicherlich gilt letzteres von dem Antiphymatol.

5. Die Wartung der Impflinge kann Gefahren für die Menschen insotern einschliessen, als die den Tieren eingespritzten freien virulenten Tuberkelbazillen mit den Exkreten ausgeschieden werden, oder beim Misslingen der intravenösen Injektion in die Subkutis gelangen und hier einen tuberkulösen Abszess erzeugen, der im weiteren Verlauf aufbrechen kann und einen virulente Menschen- 
tuberkelbazillen haltigen Eiter entleert. Die ausgeschiedenen Tuberkelbazillen können in verschiedener Weise ihren Weg in den Menschen (Stallpersonal) finden. Diese Gefahr liegt vorwiegend beim 'T a uruman, weniger beim Borovakzin und nicht bei dem Antiphymatol vor.

6. Der Genus v on Fleisch der Impflinge kann nur dann Gefahren für die menschliche Gesundheit einschliessen, wenn die Impfstoffe virulente Tuberkelbazillen enthalten. Das Antiphymatol ist auch hier ungefährlich, das gleiche gilt von dem $\mathrm{Heymansschen}$ Imp fst off, soweit die Schilfsäckchen vollkommen dicht sind. Die Bovovakzin - und Taurumanbazillen können sich längere Zeit im Rinderkörper lebend und virulent erhalten. Um die menschliche Gesundheit durch den Genuss von Tieren, die mit Bovovakzin und Tau ruman geimpft sind, nicht zu gefährden, empfiehlt es sich, bei der Fleischbeschau der mit den genannten Impfstoffen behandelten Tiere nach folgenden Vorschlägen des Kais. Gesundheitsamtes zu verfahren.

a) Lunge und Herz von mit lebenden Tuberkelbazillen immunisierten Rindern sind 10 Monate lang nach der Impfung untauglich.

b) Finden sich Veränderungen an der Impfstelle, so ist die Impfstelle und ihre Cmgebung bis einschliesslich der zugehörigen Lymphdrüsen untauglich.

c) Der ganze Tierkörper mit Ausnahme von Lunge und Herz ist innerhalb der ersten 4 Monate nach der Impfung bedingt tauglich.

Hinsichtlich des Fleisches der mit dem Antiphymatol behandelten Tiere sind Verkehrsbeschränkungen nicht geboten.

7. Der Genuss von Milch der Impflinge kann in gleicher Weise, wie dies hinsichtlich des Fleisches betont worden ist, nur dann die menschliche Gesundbeit bedrohen, wenn die Impfstoffe virulente Tuberkelbazillen enthalten. Das Antiphymatol ist hinsichtlich des Milchgenusses völlig ungefährlich, sogar dann, wenn es milchenden Tieren eingeimpft wird. Das gleiche gilt auch von dem Heymansschen Impfstoff, solange die Schilfsäckchen vollkommen dicht sind. Die virulenten Bovorakzin- und Taurumanbazillen können sehr leicht mit der Milch ausgeschieden werden, wenn sie entgegen den Vorschriften älteren weiblichen Rindern eingespritzt werden. Inwieweit. virulente Menschentuberkelbazillen dann mit der Milch ausgeschieden werden können, wenn die Tiere mit dem Tauruman und Bovovakzin im vorschriftsmässigen Alter von höchstens $1 / 4 \mathrm{Jahr}$ erstmalig geimpft werden, bedarf noch weiterer Untersuchung, wobei auch solche Tiere mit zu berücksichtigen sind, bei denen der Impfstoff entgegen der Vorschrift in die Subkutis gelangt ist. 
8. Die Impfiverluste betragen bei der Behring schen Methode $3 / 4 \%$; bei der Koch-Schützschen Taurumanimpfung sind sie noch erheblicher, dagegen sind Verluste im Anschluss an ca. 30000 Impfungen nach Heymans und Klimmer nicht beobachtet worden. Zuweilen sind durch die Bovakzin- und Taurumanimprungen Augen- und Gelenktuberkulose erzengt worden.

9. Die Wirksamkeit des v. Behringschen und KochSchützschen Schutzimpfverfahrens ist im Hinblick auf die nur etwa einjährige Dauer des Impfschutzes und die aus sanitären Gründen etc. gegebene Unmöglichkeit, den Impfschutz durch Nachimpfungen zu verlängern, als ungenügend zu bezeichnen. Das Heymanssche Verfahren lässt sich zurzeit noch nicht hinlänglich sicher beurteilen.

Das Klimmersche Verfahren hat als Prophylaktikum bisher im allgemeinen den Anforderungen der Praxis entsprochen. Bei tuberkulösen, der Impfung unterzogenen Rindern ist bisher bei der 1-4 Jahre später vorgenommenen Schlachtung beobachtet worden, dass der beschränkte tuberkulöse Prozess zum Stillstand kommt, der tuberkulöse Herd abkapselt und vielfach verkalkt, ohne dass neue Herde auftreten.

10. Durch weitere, umfangreiche Versuche ist für die Beurteilung der Wirksamkeit der Impfrerfahren weiteres Beobachtungsmaterial zu sammeln und namentlich gilt dies von den beiden zuletzt genannten Verfahren.

\section{Anhang:}

\section{Vorschriften für die Durchfïhrung des Tuberkulose-Impf- verfahrens mit Hilfe von Antiphymatol ${ }^{1}$;}

1. Das Tuberkulose-Impfverfahren mit Hilfe von Antiphymatol nach Professor Klimmer eignete sich für Rinder aller Altersklassen und wird sowohl als Schutzimpfung tuberkulosefreier, als auch als Heilimpfung tuberkulöser Rinder durchgeführt. Der Heilungsvorgang führt bei ganz frischen Prozessen zur völligen Rückbildung, bei bereits vorgeschritteneren zur Abkapselung.

2. Als Impfstoff (Antiphymatol) dient eine gebrauchsfertig bezogene Anfschwemmung von avirulenten Tuberkelbazillen, also von Baliterien, die bei sachgemässer Anwendung für Menschen und Impflinge gleich ungefährlich sind.

1) Zu beziehen bei Humann und Teisler, Dohna bei Dresden. 
3. Das Antiphymatol wird in eingeschmolzenen Glasröhrchen gebrauchsfertig abgegeben. Der Impfstoff ist möglichst frisch, auf jeden Fall innerhalb der auf jeder einzelnen Dosis angegebenen Zeit (ca. 6 Worhen) zu gebrauchen und bis zur Verwendung kühl und dunkel aufzubewaluren. Vor dem Gebrauche ist der Impfstoff kräftig durchzuschütteln. Hierauf wird der engere Teil des Glasröhrchens mit einer kantigen Feile angeschnitten und abgebrochen. Der Impfstoff eines geöffneten Röhrchens ist noch am selbigen Tage zu verbrauchen.

4. Das Antiphymatol wird aus dem geöffneten Röhrchen in eine Pravazsche Spritze aufgesogen. Die Spritze, welche möglichst nur für die Impfungen mit diesem T'uberkulose-Impfstoff zu verwenden ist, muss stets sauber gehalten und vor und nach dem Gebrauche am besten durch Auskochen oder sonst durch Ausspülen mit einer Desinfektionsflüssigkeit (einpromillige Sublimatlösung, zweiprozentige Lysollösung etc.) und hierauf mit abgekochtem Wasser gereinigt werden.

๖. Die Impflosis beträgt $5 \mathrm{ccm}$ des Antiphymatols.

6. Das Antiphymatol wird den Rindern unter die Haut gespritzt. Als Impfstelle eignet sich vornehmlich die Halsseite. Eine besordere Behandlung der Impfstelle vor oder nach der Einspritzung ist nicht notwendig.

7. Die Schutzimpfung ist:

a) ein Vierteljahr nach der ersten Einspritzung und

b) alljährlich einmal zu wiederholen.

Besteht der Verdacht, dass der Impiling schon vor der Schutzimpfung an Tuberkulose erkrankt ist, so ist die Heilimpfung zweckmässigerweise im ersten Jahr in einvierteljährigen Zwischenpausen viermal vorzunehmen und ebenfalls alljährlich einmal zu wiederholen.

8. Zu Beginn des Tuberkulose-Impfverfahrens in einem Bestande sind die Rinder der leicht durchzuführenden A u g e n p r ob e (Ophthalmoreaktion) mit „Phymatin“ zu unterziehen. Die nicht reagierenden Tiere sind von den reagierenden zu trennen, d. h. in geschlossenen Reihen aufzustellen. Stehen die Rinder mit den Köpfen einander gegenüber, so stellt man den nicht reagierenden Tieren, wenn möglich, auch nicht reagierende gegenüber. Die Kälber sind, wenn sie nicht mit Phymatin vorgeprüft werden, insgesamt, andernialls nur soweit sie nicht reagieren, der gesunden, nicht reagierenden Abteilung anzureihen. Tiere mit vorgeschrittener Lungentuberkulose und eutertuberkulöse Kühe sind baldigst abzuschlachten.

9. Es empfiehlt sich, die Impflinge vor und etwa 2 Monate nach der ersten Schutzimpfung möglichst vor einer Tuberkuloseansteckung 
zu bewahren. In dieser Richtung kommt bei der jungen Aufzucht in erster Linie die Ernährung mit einwandfreier Milch in Frage.

10. Den Kälbern ist entwerler Milch tuberkulosefreier Kühe (zum mindesten solcher Kühe, welche frei von Eatertuberkulose und sonstiger klinisch feststellbarer Tuberkulose sind) oder abgekochte bezw. pasteurisierte Milch zu geben. Die Pasteurisierung kommt namentlich bei der Magermilch aus Sammelmolkereien in Frage. Die Bekömmlichkeit und Ausnutzung der abgekochten bezw. pasteurisierten Milch wird durch Zusatz von 2 Gramm Kochsalz auf' 1 Liter Milch wesentlich gebessert. Sind diese Massnahmen nicht durchfiihrbar, so sollte jedes Kalb nur mit der Milch einer bestimmten Kuh und nicht mit der Mischmilch mehrerer Kühe ernährt werden.

11. Den schutzgeimpften Tieren ist nach Möglichkeit Aufenthalt und Bewegung im Freien zu geben. Bei Weidegang ist auf eine ausreichende und kräftige Ernährung der jungen Aufzucht, namentlich bei dürftigem Graswuchs und bergigem Gelände, zu achten.

12. Im Stalle lässt man die schutzgeimpften Tiere nach Möglichkeit in Boxen frei herumlaufen. Beim Anbinden stellt man die Inplinge direkt hintereinander auf. Stehen die Rinder in doppelten Reihen mit den Köplen gegenüber, so sind den schutzgeimpften Rindern nach Möglichkeit auch schutzgeimpfte Tiere gegenüber zu stellen. 